\title{
Askin Tumor: A Case Report of Primitive Neuroectodermal Tumors of the Chest Wall - Computed Tomography Findings
}

\author{
${ }^{1}$ Namita S Chandak, ${ }^{2}$ Shivali Kashikar, ${ }^{3}$ Shiba Khan, ${ }^{4}$ Saherish Khan
}

\begin{abstract}
Askin tumor is a rare malignant neoplasm in the thoracopulmonary region. Its diagnostic approach is complex and requires a multidisciplinary team. Given the rarity of this entity, we report a case of Askin's tumor.
\end{abstract}

How to cite this article: Chandak NS, Kashikar S, Khan S, Khan S. Askin Tumor: A Case Report of Primitive Neuroectodermal Tumors of the Chest Wall - Computed Tomography Findings. Int J Recent Surg Med Sci 2016;2(2):111-113.

Keywords: Askin's tumor, Chest wall tumor, Ewing sarcoma.

Source of support: Nil

Conflict of interest: None

\section{INTRODUCTION}

Ewing sarcoma (ES) and peripheral primitive neuroectodermal tumor (PNET) (previously called peripheral neuroepithelioma) were originally described in the early 1900s as distinct clinicopathologic entities. They are rare malignant small round cell tumors that arise from the primitive nerve cells of the nervous system, but they can also occur outside the central nervous system in the chest wall, pelvis, and extremities. ${ }^{1}$ It develops from the soft tissues of the chest wall, particularly in the paravertebral region.

By race, Caucasians have the highest incidence (0.155), followed by Asians/Pacific Islanders (0.082), and African-Americans (0.017). There is a ninefold difference in incidence between Caucasians and African-Americans $(p<0.001)$. The incidence of ES increased over the past three decades among Caucasians $(\mathrm{p}<0.05)$. Survival was not impacted by race. ${ }^{2}$

\footnotetext{
${ }^{1,3,4}$ Resident, ${ }^{2}$ Professor

${ }^{1}$ Department of Radiodiagnosis, Datta Meghe Institute of Medical Sciences, Wardha, Maharashtra, India

${ }^{2-4}$ Department of Radiodiagnosis, Jawaharlal Nehru Medical College, Wardha, Maharashtra, India

Corresponding Author: NamitaSChandak, Resident, Department of Radiodiagnosis, Datta Meghe Institute of Medical Sciences Wardha, Maharashtra, India, Phone: +919881675449, e-mail: namitachandak1@gmail.com
}

\section{CASE REPORT}

A 11-year-old male presented with a 2 months' history of cough, weight loss, and pain in the right side of the chest. Physical examination revealed a slightly tender soft mass in the right chest wall and decreased breath sound in the right lung without any other abnormality.

Computed tomography (CT) chest scanogram revealed a large opacity in the right hemithorax. For detailed evaluation, a contrast-enhanced CT scan was justified. The CT revealed a large, heterogeneously enhancing right chest wall mass of size $15 \times 14 \mathrm{~cm}$ in the right hemithorax causing compression atelectasis of right lower lobe, middle lobe, and posterior segment of right upper lobe with diffuse scattered fluid attenuating areas within. The mass was displacing the mediastinum toward the left side (Figs 1 to 4). Computed tomographyguided fine needle aspiration cytology (FNAC) from right lung mass showed small round cells in a branching perivascular pattern in pseudorosettes, suggesting malignant small round cell tumor of extraskeletal ES, possibly Askin tumor.

\section{DISCUSSION}

Ewing sarcoma and peripheral PNET were originally described as distinct clinicopathologic entities: in 1918, Stout described a tumor of the ulnar nerve composed of small round cells focally arranged as rosettes; this entity was subsequently designated neuroepithelioma and then PNET. ${ }^{3}$ Later, in 1921, ES was described as an undifferentiated tumor involving the diaphysis of long bones. It was also reported to arise in soft tissue (extraosseous ES). ${ }^{4}$ Nevertheless, it has become clear, over the last three decades, that these entities comprise the same spectrum of neoplastic diseases known as the ES family of tumors, which also includes malignant small cell tumor of the chest wall and atypical ES. Ewing sarcoma of the chest wall was originally reported by Askin et $\mathrm{al}^{1}$ in 1979 in 20 white children and adolescents (average age, 14 years). Since then, PNETs within the thoracopulmonary region have been termed Askin tumors. This is a rare disease in the pediatric group, and even rarer in adults. Caucasian ethnic group is clearly predominant. ${ }^{5}$ 


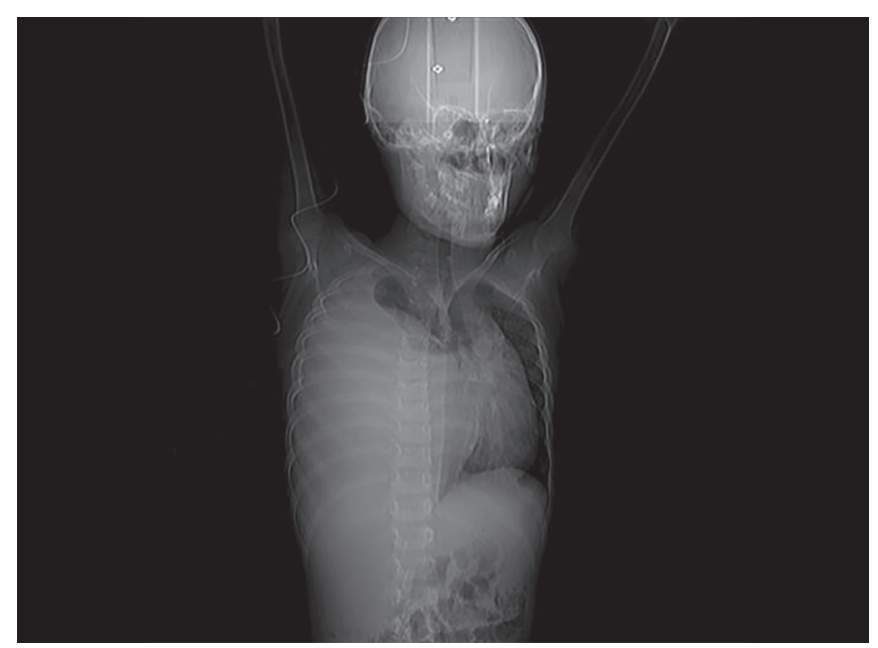

Fig. 1: Computed tomography scanogram showing homogeneous opacity in the right hemithorax displacing the mediastinum toward the left with collapse of right lung

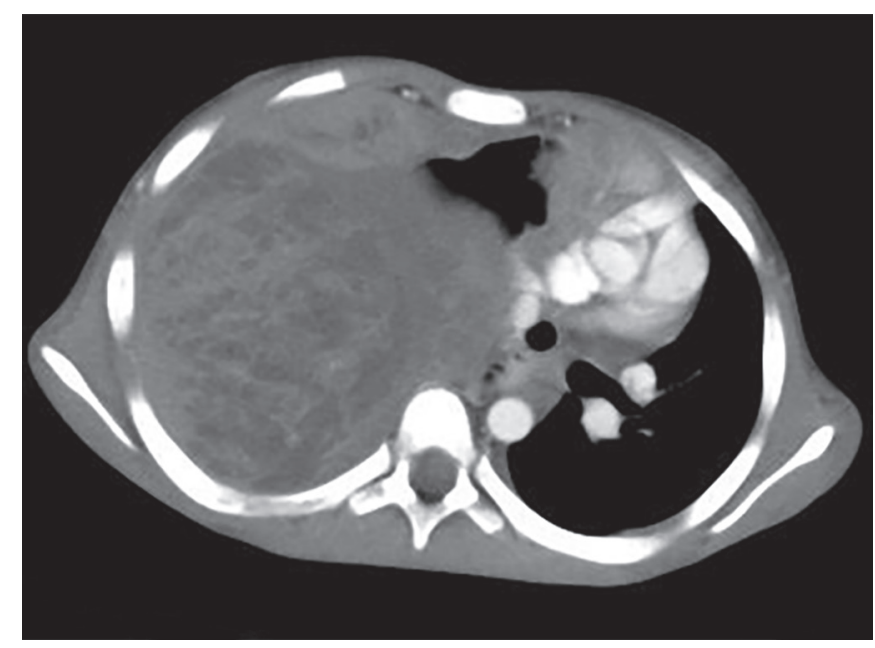

Fig. 3: Axial contrast-enhanced CT shows a large heterogeneously enhancing right chest wall mass of size $15 \times 14 \mathrm{~cm}$ in the right hemithorax causing compression atelectasis of right lung with cystic areas within. The mass is causing displacement of mediastinum toward the left side

The most important role of the CT scan is to confirm the presence of a solid chest wall lesion and to demonstrate their possible intrathoracic extension and/or direct lung or mediastinal invasion. ${ }^{5,6}$

Askin tumors on magnetic resonance imaging are described as heterogeneous soft tissue masses with a moderate to high signal intensity on T1-weighted images (greater than skeletal muscle) and high signal intensity on T2-weighted images.

Differential diagnosis of imaging with chest wall mass occurring in children and adolescents should include neuroblastoma, rhabdomyosarcoma, non-Hodgkin lymphoma, and Langerhans cell histiocytosis. ${ }^{78}$

\section{CONCLUSION}

The described radiologic features of Askin tumors may be useful in deciding when to include this entity in the

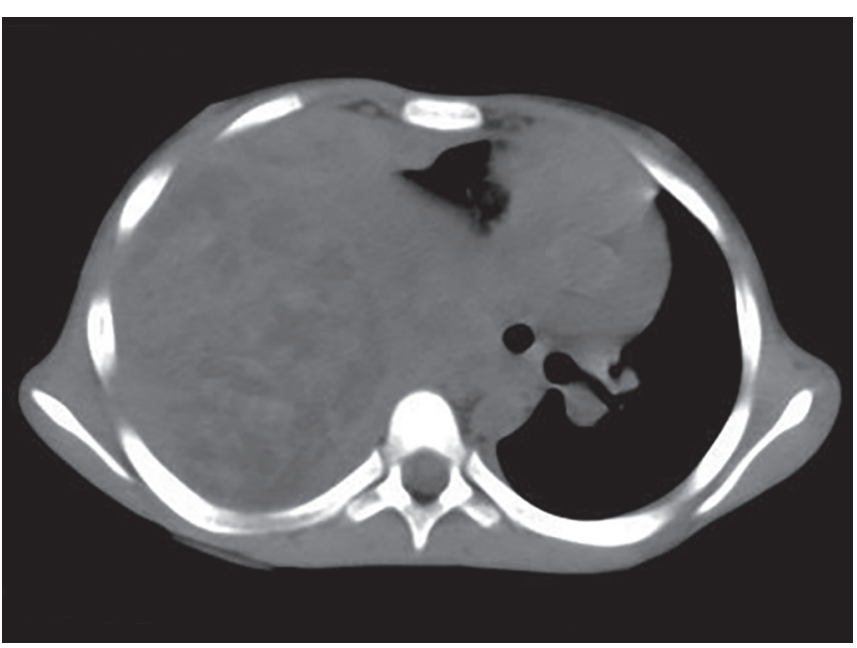

Fig. 2: Plain CT thorax reveals large heterogeneous right chest wall mass displacing the mediastinum

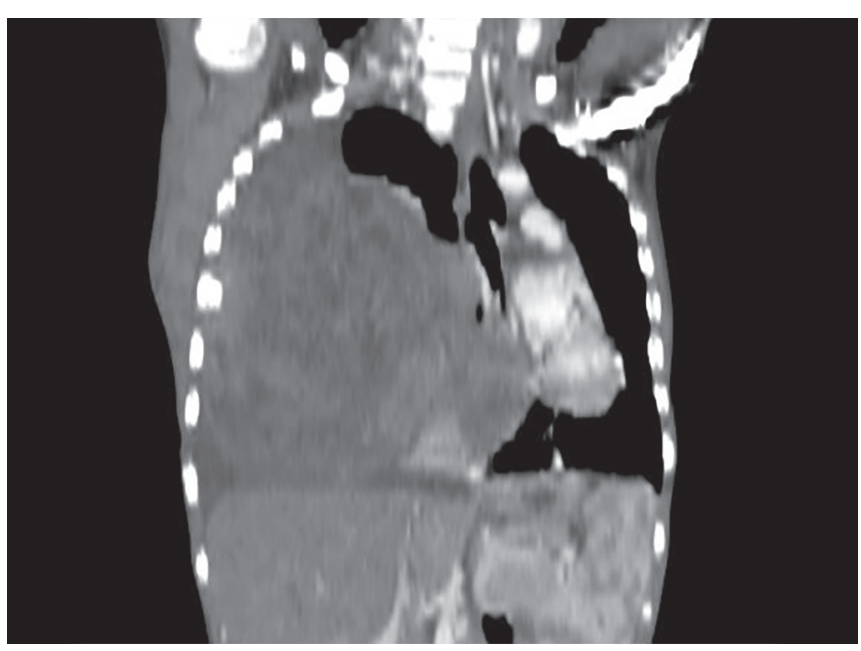

Fig. 4: Coronal contrast-enhanced CT reveals the large right chest wall mass

differential diagnosis of childhood chest wall masses. Areas of hemorrhage and necrosis in the large tumors are responsible for their heterogeneous appearance on CT scan images. Computed tomography scanning is preferred for detecting pulmonary, osseous, and hepatic metastases. ${ }^{7}$

\section{REFERENCES}

1. Askin FB, Rosai J, Sibley RK, Dehner LP, McAlister WH. Malignant small cell tumour of the thoracopulmonary region in childhood: a distinctive clinicopathologic entity of uncertain histogenesis. Cancer 1979 Jun;43(6): 2438-2451.

2. Jawad MU, Cheung MC, Min ES, Schneiderbauer MM, Koniaris LG, Scully SP. Ewing sarcoma demonstrates racial disparities in incidence-related and sex-related differences in outcome. Cancer 2009 Aug;115(15):3526-3536.

3. Jaffe R, Santamaria M, Yunis EJ, Tannery NH, Agostini RM Jr, Medina J, Goodman M. The neuroectodermal tumor of bone. Am J Surg Pathol 1984 Dec;8(12):885-898. 
4. Angervall L, Enzinger FM. Extraskeletal neoplasm resembling Ewing's sarcoma. Cancer 1975 Jul;36(1):240-251.

5. Laskar S, Nair C, Mallik S, Bahl G, Pai S, Shet T, Gupta T, Arora B, Bakshi A, Pramesh CS, et al. Prognostic factors and outcome in Askin-Rosai tumor: a review of 104 patients. Int J Radiat Oncol Biol Phys 2011 Jan;79(1):202-207.

6. Sabati JM, Franquet T, Parellada JA, Monill JM, Oliva E. Malignant neuroectodermal tumour of the chest wall (Askin tumour): CT and MR findings in eight patients. Clin Radiol 1994 Sep;49(9):634-638.

7. Winer-Muram HT, Kaufman WH, GronemeyerSA, Jennings SG. Primitive neuroectodermal tumors of the chest wall (Askin tumors): CT and MR findings. Am J Res 1985 Aug;145(2):517-520.

8. Xu Q, Xu K, Yang C, Zhang X, Meng Y, Quan Q. Askin tumor: four case reports and a review of the literature. Cancer Imaging 2011 Nov;11(1):184-188. 